\title{
Spectrum-based estimation method for peak structural responses of a SDOF system pounding against rigid structures
}

\author{
B. T. Tran \& K. Kasai \\ Structural Engineering Research Center, Tokyo Institute of Technology, \\ Japan
}

\begin{abstract}
A spectrum-based method is proposed for predicting peak responses of a SDOF system that pounds against adjacent rigid structure(s) during an earthquake. Pounding is considered to occur on either one side or two sides of the system. The contact element is simulated by the Kelvin body combining a spring and a dashpot in parallel. Based on free vibration and harmonic vibration both involving pounding, the equivalent period and damping of the SDOF system are obtained and used to predict peak displacements as well as collision force. The accuracy of the proposed method is demonstrated via extensive numerical experiments over a variety of combinations of system properties, contact element properties, separation distances, and earthquakes.

Keywords: relative displacement, structural pounding, viscoelastic contact element, coefficient of restitution, equivalent period, equivalent damping.
\end{abstract}

\section{Introduction}

Pounding between buildings occurs when separation distance is insufficient to accommodate the peak relative displacement between them [1,2]. The collision between two buildings is typically simulated by contact element, which becomes active only when contact is detected. Several types of contact element such as linear/nonlinear spring element, and linear/nonlinear viscoelastic element have been used for pounding simulation [3-5]. The use of numerical methods for this nonlinear dynamic problem poses many difficulties and requires time-consuming efforts. Moreover, using numerical methods at the beginning of design may not be an efficient choice because of the nature of design process, where repetitive 
analyses using different ground motions are required. Therefore, an alternative method utilizing response spectra could be more suitable for practical use. The spectrum-based method would also make it possible to clarify the relationship among structural properties, earthquake characteristics, and peak responses.

The objective of our research, therefore, is to propose simplified methods for estimating peak responses of buildings subjected to seismic pounding. This paper will concentrate on the fundamental pounding problem, where a SDOF system pounds against adjacent rigid structure(s) located on its side(s) and viscoelastic contact element is used for pounding simulation. Equivalent period and damping are derived, then combined with elastic response spectra to get peak responses. The proposed method is simple but accurate enough for practical application.

\section{Modelling of pounding problem}

Consider a SDOF system with mass $m$, stiffness $k$, and viscous damping coefficient $c$. It pounds against adjacent rigid wall(s) when absolute displacement $|u(t)|$ exceeds either separation distance $s^{+}(>0)$ on the right side, or $s^{-}(>0)$ on the left side. Viscoelastic contact elements (fig. 1a) are characterized as parallel combination of linear spring stiffness $\left(k_{s}^{+}\right.$or $\left.k_{s}^{-}\right)$and dashpot $\left(c_{s}^{+}\right.$or $\left.c_{s}^{-}\right)$.
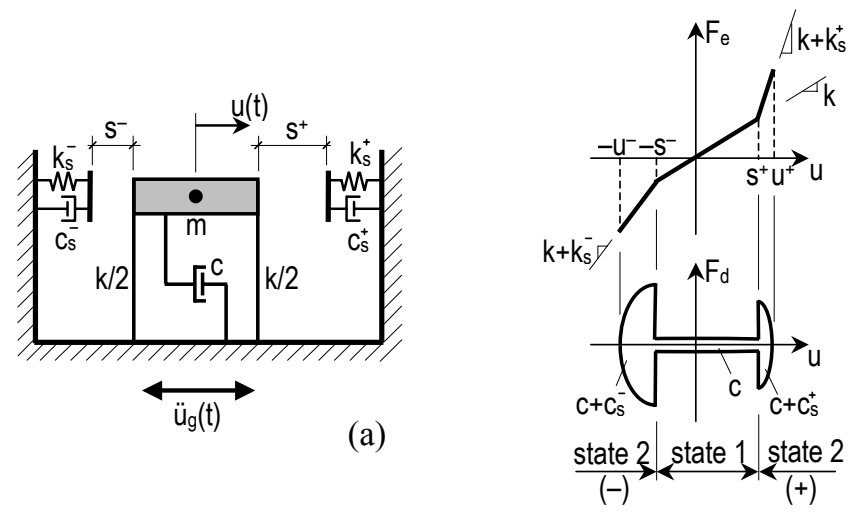

Figure 1: Pounding of a SDOF system against rigid structures: (a) Modeling, (b) Force-displacement relations.

The system is considered to be in state 1 when not in contact and state 2 when in contact with the wall. Elastic force $F_{e}$ and damping force $F_{d}$ are governed by displacement $u$ (fig. $1 \mathrm{~b}$ ) and velocity $\dot{u}$ as follows:

$$
\begin{aligned}
& F_{e}=k u, \quad F_{d}=c \dot{u} \quad\left(\text { state } 1:-s^{-} \leq u \leq s^{+}\right) \\
& F_{e}=k u+k_{s}^{+}\left(u-s^{+}\right), F_{d}=\left(c+c_{s}^{+}\right) \dot{u} \quad\left(\text { state } 2: u>s^{+}\right) \\
& F_{e}=k u+k_{s}^{-}\left(u+s^{-}\right), F_{d}=\left(c+c_{s}^{-}\right) \dot{u} \quad\left(\text { state } 2: u<-s^{-}\right)
\end{aligned}
$$


Clearly, stiffness and damping are added by contact element when the state changes from 1 to 2 , hereby we define the stiffness increase ratio $\kappa^{+}$and $\kappa^{-}$, as well as damping increase ratio $\zeta^{+}$and $\zeta^{-}$, eqns. (2) and (3).

$$
\begin{array}{ll}
\kappa^{+}=1+k_{s}^{+} / k, & \zeta^{+}=1+c_{s}^{+} / c \\
\kappa^{-}=1+k_{s}^{-} / k, & \zeta^{-}=1+c_{s}^{-} / c
\end{array}
$$

Let call vibration frequency in state 1 as no-pounding vibration frequency $\omega_{n p}$, and those at state 2 as pounding vibration frequencies $\omega_{p d}^{+}$and $\omega_{p d}^{-}$. The vibration frequencies and damping ratios are related as follows:

$$
\begin{aligned}
& \omega_{p d}^{+}=\sqrt{\left(k+k_{s}^{+}\right) / m}=\sqrt{\kappa^{+}} \omega_{n p}, \quad \xi_{p d}^{+}=\frac{c+c_{s}^{+}}{2 m \omega_{p d}^{+}}=\frac{\zeta^{+}}{\sqrt{\kappa^{+}}} \xi_{n p} \\
& \omega_{p d}^{-}=\sqrt{\left(k+k_{s}^{-}\right) / m}=\sqrt{\kappa^{-}} \omega_{n p}, \quad \xi_{p d}^{-}=\frac{c+c_{s}^{-}}{2 m \omega_{p d}^{-}}=\frac{\zeta^{-}}{\sqrt{\kappa^{-}}} \xi_{n p}
\end{aligned}
$$

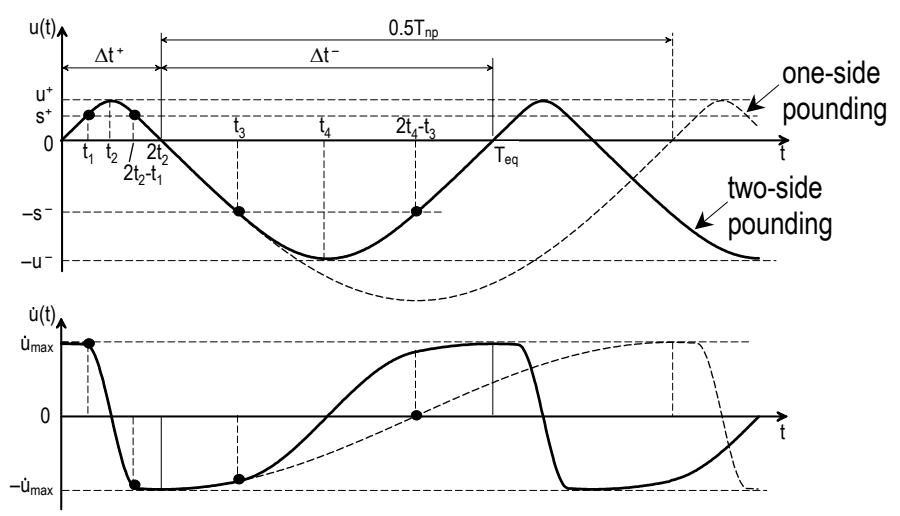

Figure 2: Time history of undamped free vibration.

\section{Free vibration with pounding}

\subsection{Time history of undamped free vibration pounding response}

Considering initial condition $u=0$ and $\dot{u}=\dot{u}_{\max }$, where $\dot{u}_{\max }>\omega_{n p} s^{+}$and $\omega_{n p} s^{-}$ so that pounding will occur on both sides. By a similar process [6], we can obtain a) $0 \leq t \leq t_{1}$ (state 1 , see also fig. 2):

$$
u(t)=\left(\dot{u}_{\max } / \omega_{n p}\right) \sin \omega_{n p} t, t_{1}=\left\{\sin ^{-1}\left(\omega_{n p} s^{+} / \dot{u}_{\max }\right)\right\} / \omega_{n p}
$$

b) $t_{1}<t<2 t_{2}-t_{1}$ (state 2$)$ :

$$
u(t)=s^{+}+\left(s^{+} / \kappa^{+}\right)\left\{-1+\cos \omega_{p d}^{+}\left(t-t_{1}\right)\right\}+\left\{\dot{u}\left(t_{1}\right) / \omega_{p d}^{+}\right\} \sin \omega_{p d}^{+}\left(t-t_{1}\right)
$$




$$
t_{2}=t_{1}+\left(\omega_{n p} \sqrt{\kappa^{+}}\right)^{-1} \tan ^{-1} \sqrt{\kappa^{+}\left\{\left(\dot{u}_{\max } / \omega_{n p} / s^{+}\right)^{2}-1\right\}}
$$

c) $2 t_{2}-t_{1} \leq t \leq t_{3}($ state 1$)$ :

$$
u(t)=\left(-\dot{u}_{\max } / \omega_{n p}\right) \sin \omega_{n p}\left(t-2 t_{2}\right), t_{3}=\left\{\sin ^{-1}\left(\omega_{n p} s^{-} / \dot{u}_{\max }\right)\right\} / \omega_{n p}+2 t_{2}
$$

d) $t_{3}<t<2 t_{4}-t_{3}($ state 2$)$ :

$$
\begin{gathered}
u(t)=-s^{-}-\left(s^{-} / \kappa^{-}\right)\left\{-1+\cos \omega_{p d}^{-}\left(t-t_{3}\right)\right\}+\left\{\dot{u}\left(t_{3}\right) / \omega_{p d}^{-}\right\} \sin \omega_{p d}^{-}\left(t-t_{3}\right) \\
t_{4}=t_{3}+\left(\omega_{n p} \sqrt{\kappa^{-}}\right)^{-1} \tan ^{-1} \sqrt{\kappa^{-}\left\{\left(\dot{u}_{\max } / \omega_{n p} / s^{+}\right)^{2}-1\right\}}
\end{gathered}
$$

For one-side pounding at right, negative state 2 displacement, eqn. (9a), does not exist. Thus, time $t_{4}$ is given by $\mathrm{d} u(t) / \mathrm{d} t=0$ of eqn. (8a), and $t_{4}=2 t_{2}+T_{n p} / 4$ instead of eqn. (9b) is obtained (fig. 2). In contrast, for one-side pounding at left, time $t_{2}$ is given by $\mathrm{d} u(t) / \mathrm{d} t=0$ of eqn. (6a), and $t_{2}=T_{n p} / 4$ instead of eqn. (7b).

\subsection{Peak displacement and equivalent vibration period}

We defined (fig. 2) $u^{+}$and $u^{-}$as absolute values of positive and negative peak displacements, respectively. $u^{+}$can be expressed with $\dot{u}_{\max }$ by substituting eqn. (7b) into eqn. (7a), similarly for $u^{-}$by substituting eqn. (9b) into eqn. (9a), then

$$
\begin{aligned}
& u^{+}=s^{+}\left[1-1 / \kappa^{+}+\sqrt{\left\{1 / \kappa^{+}-1+\left(\dot{u}_{\max } / \omega_{n p} / s^{+}\right)^{2}\right\} / \kappa^{+}}\right] \\
& u^{-}=s^{-}\left[1-1 / \kappa^{-}+\sqrt{\left\{1 / \kappa^{-}-1+\left(\dot{u}_{\max } / \omega_{n p} / s^{-}\right)^{2}\right\} / \kappa^{-}}\right]
\end{aligned}
$$

Rewriting eqn. (10):

$$
\begin{aligned}
\dot{u}_{\max } & =\omega_{n p} u^{+} \sqrt{1+\left(1-s^{+} / u^{+}\right)^{2}\left(\kappa^{+}-1\right)} \\
& =\omega_{n p} u^{-} \sqrt{1+\left(1-s^{-} / u^{-}\right)^{2}\left(\kappa^{-}-1\right)}
\end{aligned}
$$

From eqns. (7b) and (9b), durations of responses per cycle showing positive and negative displacements $\Delta t^{+}\left(=2 t_{2}\right)$ and $\Delta t^{-}\left(=2\left(t_{4}-2 t_{2}\right)\right)$ are obtained as follows:

$$
\begin{aligned}
& \Delta t^{+}=\left(T_{n p} / \pi\right)\left[\sin ^{-1}\left(\omega_{n p} s^{+} / \dot{u}_{\max }\right)+\left(\kappa^{+}\right)^{-0.5} \tan ^{-1} \sqrt{\kappa^{+}\left\{\left[\dot{u}_{\max } /\left(\omega_{n p} s^{+}\right)\right]^{2}-1\right\}}\right] \\
& \Delta t^{-}=\left(T_{n p} / \pi\right)\left[\sin ^{-1}\left(\omega_{n p} s^{-} / \dot{u}_{\max }\right)+\left(\kappa^{-}\right)^{-0.5} \tan ^{-1} \sqrt{\kappa^{-}\left\{\left[\dot{u}_{\max } /\left(\omega_{n p} s^{-}\right)\right]^{2}-1\right\}}\right]
\end{aligned}
$$

where $T_{n p}$ is no-pounding period. Eqn. (12) indicates that $\Delta t^{+}, \Delta t^{-}$depend on $\dot{u}_{\max }, s^{+}$, and $s^{-}$. Thus, equivalent vibration period $T_{e q}$ can be given as follows:

$$
T_{e q}=\Delta t^{+}+\Delta t^{-} \quad \text { (two-side pounding) }
$$

In case of one-side pounding at right, for example, $u^{+}$is given by eqn. (10a), and since pounding does not occur at left: $u^{-}=\dot{u}_{\max } / \omega_{n p}$ by setting $\kappa^{-}=1$ in eqn. (10b). Duration $\Delta t^{+}$and $\Delta t^{-}$are obtained in an analogous manner, therefore 


$$
\begin{array}{ll}
T_{e q}=\Delta t^{+}+0.5 T_{n p} & \text { (one - side pounding at right) } \\
T_{e q}=\Delta t^{-}+0.5 T_{n p} & \text { (one }- \text { side pounding at left) }
\end{array}
$$
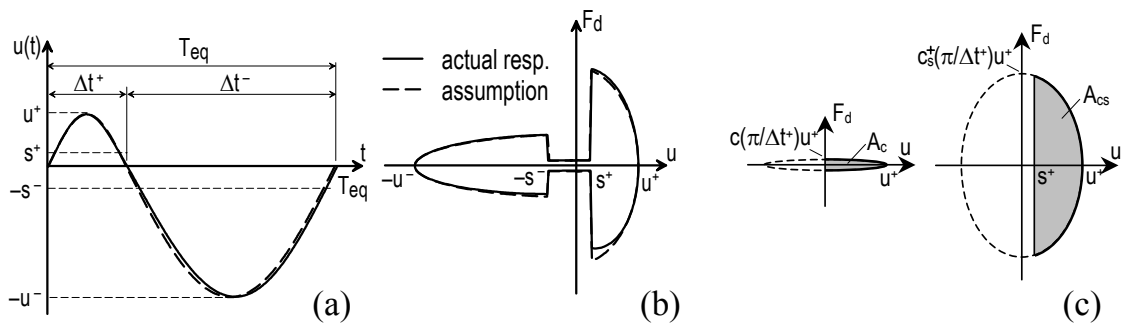

(b)

(c)

Figure 3: $\quad$ Steady state response of two-side pounding: (a) time history, (b) $F_{d}-u$ relationship, (c) calculation of dissipated energy.

\section{Harmonic vibration with pounding}

\subsection{Equivalent damping ratio}

The equivalent damping ratio $\xi_{\text {eq }}$ is normally based on the steady state response of a system to harmonic force at exciting frequency equal to the natural frequency of the system. To apply for current problem, the equivalent period $T_{e q}$ derived in previous section is used as natural period of the pounding system, and the equation of motion under considering harmonic force can be written as

$$
m \ddot{u}+F_{d}(\dot{u}(t))+F_{e}(u(t))=-m \ddot{u} \ddot{g}_{0} \sin \left(2 \pi t / T_{e q}\right)
$$

After a number of numerical checks (fig. 3a, b), the steady state $u(t)$ in a cycle of vibration is approximated by two distinct half-sinusoidal curves with vibration frequencies $\left(\pi / \Delta t^{+}\right)$and $(\pi / \Delta t)$ for each side (fig. 3a), respectively. Consequently, energy $E_{D}^{+}$dissipated while $u(t)>0$ can be calculated as the summation of dissipated energies by system damping $c$ (area $A_{c}$ ) and contact dashpot $c_{s}^{+}$(area $A_{c s}$ ), see fig. 3c. Similarly for dissipated energy $E_{D}^{-}$while $u(t)<0$ and we obtain

$$
\begin{aligned}
& E_{D}^{+}=c \frac{\left(\pi u^{+}\right)^{2}}{2 \Delta t^{+}}\left[1+\frac{2 c_{s}^{+}}{\pi c}\left\{\cos ^{-1} \frac{s^{+}}{u^{+}}-\frac{s^{+}}{u^{+}} \sqrt{1-\left(s^{+} / u^{+}\right)^{2}}\right\}\right] \\
& E_{D}^{-}=c \frac{\left(\pi u^{-}\right)^{2}}{2 \Delta t^{-}}\left[1+\frac{2 c_{s}^{-}}{\pi c}\left\{\cos ^{-1} \frac{s^{-}}{u^{-}}-\frac{s^{-}}{u^{-}} \sqrt{1-\left(s^{-} / u^{-}\right)^{2}}\right\}\right]
\end{aligned}
$$

The strain energies $E_{S}$ on each side are assumed to be equal and calculated using the stiffness and peak displacements (fig. 1b) 


$$
\begin{aligned}
E_{S} & =\frac{1}{2} k\left[1+\left(\kappa^{+}-1\right)\left(1-s^{+} / u^{+}\right)^{2}\right]\left(u^{+}\right)^{2} \\
& =\frac{1}{2} k\left[1+\left(\kappa^{-}-1\right)\left(1-s^{-} / u^{-}\right)^{2}\right]\left(u^{-}\right)^{2}
\end{aligned}
$$

Based on these,

$$
\begin{aligned}
& \xi_{e q}=\Delta \xi^{+}+\Delta \xi^{-} \quad \text { (two-side pounding) } \\
& \Delta \xi^{+}=E_{D}^{+} /\left(4 \pi E_{S}\right), \Delta \xi^{-}=E_{D}^{-} /\left(4 \pi E_{S}\right)
\end{aligned}
$$

If there is only one-side pounding at right, set $s^{-}=u^{-}$in eqn. (16b) and use $\Delta t^{-}=$ $0.5 T_{n p}$. Similarly for one-side pounding at left, eqn. (18) becomes

$$
\begin{array}{ll}
\xi_{e q}=\Delta \xi^{+}+0.5 \xi_{n p} & \text { (one-side pounding at right) } \\
\xi_{e q}=\Delta \xi^{-}+0.5 \xi_{n p} & \text { (one - side pounding at left) }
\end{array}
$$

\subsection{Numerical experiment for harmonic vibration}

Numerical experiment is conducted using ground acceleration $\ddot{u}_{g 0} \sin (2 \pi t / T)$, where $\ddot{u}_{g 0}=0.6 g$ and $T$ is varied. We consider a system having $T_{n p}=1 \mathrm{~s}$ and $\xi_{n p}$ $=0.02$, thus peak steady state displacement of no-pounding system at resonant excitation $\left(T=T_{n p}\right)$ is $u_{n p}=\ddot{u}_{g 0} / 2 \omega_{n p}^{2} \xi_{n p}=3.72 \mathrm{~m}$. Both two-side pounding and one-side pounding are considered; for two-side pounding, separation distance is set equal on both sides, $s^{+}=s^{-}=s$. Separation distances $s=0,0.5,1$, and $4 \mathrm{~m}$ are assumed. Contact element stiffness is selected such that $\kappa=4\left(T_{p d}=0.5 \mathrm{~s}\right)$; dashpot $c_{s}$ is varied to have 6 values of $\xi_{p d}=0.01 \sim 0.52$ (fig. 4 ), respective values of coefficient of restitution $e$ [7] are also shown in fig. 4 .

Fig. 4 shows steady state displacement spectra (solid line) obtained by numerical integration. For the case of $s=4$, pounding is avoided and all the spectrum curves are identical. For the case of $s=0$, the system with two-side pounding (fig. $4 \mathrm{a}$ ) vibrates with $T_{p d}$ and $\xi_{p d}$; while the system with one-side pounding (fig. 4b), in addition to the peak of harmonic response, the peak of socalled subharmonic response appears at twice the resonant frequency of the pounding system [3]. However, the subharmonic response does not seem to have any significant effect.

\subsection{Estimation of peak displacement}

The peak displacements can be estimated by using eqn. (10) once $\dot{u}_{\text {max }}$ is known. It is assumed that $\dot{u}_{\max }$ represents the peak velocity of an equivalent linear system having $T_{e q}$ and $\xi_{e q}$, but as shown in eqns. 13 and $18, T_{e q}$ and $\xi_{e q}$ depend on $\dot{u}_{\max }$. Thus, iterations for getting $\dot{u}_{\max }$ is required and summarized below:

After setting initial values $T_{e q}^{(1)}=T_{n p}$ and $\xi_{e q}^{(1)}=\xi_{n p}$, the $i$-th iteration includes

1) Calculate $\dot{u}_{\max }^{(i)}$ using eqn. 21 for harmonic excitation, and $u^{+}$and $u^{-}$(eqn. 10).

2) Get $T_{e q}^{(i+1)}$ and $\xi_{e q}^{(i+1)}$ (eqns. 13 and 18).

3) Calculate again $\dot{u}_{\max }^{(i+1)}$ (eqn. 21), and if $\dot{u}_{\max }^{(i+1)} \approx \dot{u}_{\max }^{(i)}$, the iteration ends. 
4) Use $\dot{u}_{\max }^{(i+1)}=\left(\dot{u}_{\max }^{(i+1)}+\dot{u}_{\max }^{(i)}\right) / 2$ and go to step 1).

where

$$
\dot{u}_{\max }^{(i)}=\omega \ddot{u}_{g 0} / \sqrt{\left(\left(\omega_{e q}^{(i)}\right)^{2}-\omega^{2}\right)^{2}+\left(2 \xi_{e q}^{(i)} \omega_{e q}^{(i)} \omega\right)^{2}}
$$

In step 4), we use midpoint of the limits after each iteration so the bounds containing exact value of $\dot{u}_{\max }$ decrease by factor of two. Thus it converges fast and normally takes about 3 to 4 iterations if the tolerance limit is set to $1 \%$.

The accuracy of the method for harmonic excitation is verified as follows: $\dot{u}_{\max }$ is calculated with eqn. (11) using $u^{+}$and $u^{-}$obtained in (4.2) instead of using eqn. (21). Then steps 2), 3), and 1) are followed and the results without further iterations are compared with the time history analysis results. Estimated spectra are plotted using broken lines also in fig. 4; the estimated curves match very well with those from numerical analysis. The method can not predict subharmonic response that occurs in one-side pounding. Since such subharmonic response shows no relevant effect compared with harmonic response and it does not appear in MDOF system, its effect will not be considered again.

(a) Two-side pounding
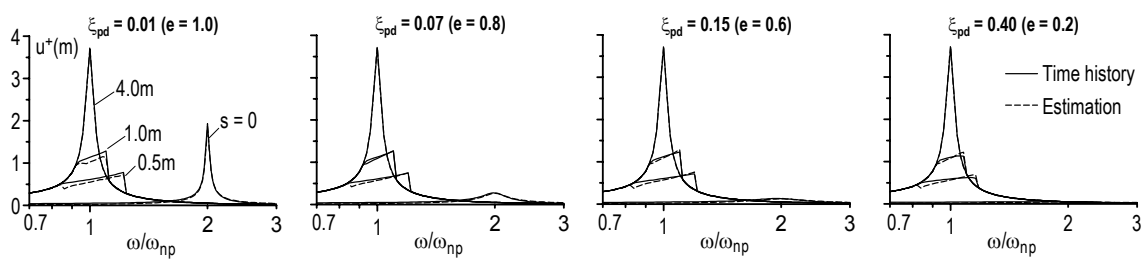

(b) One-side pounding
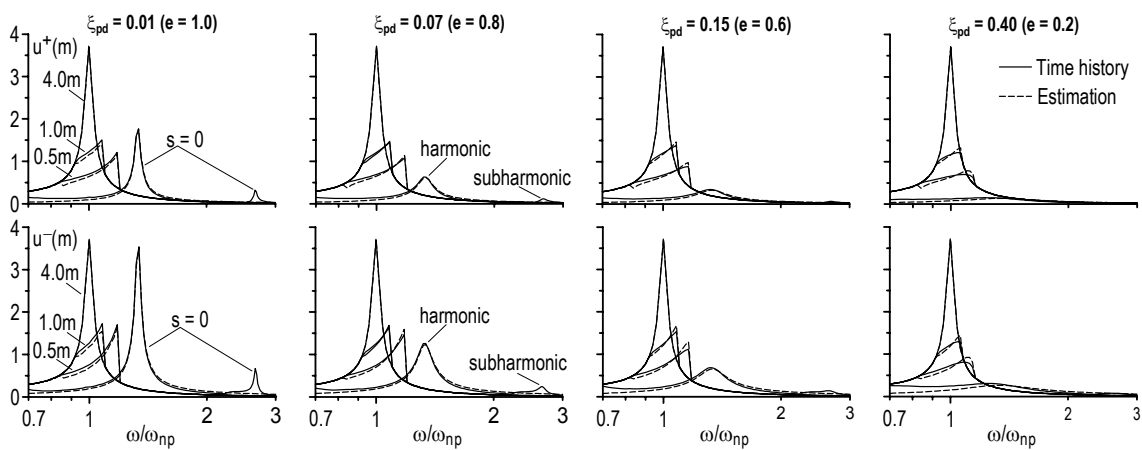

Figure 4: Displacement spectra of two-side and one-side pounding.

\section{Estimation of seismic pounding responses}

\subsection{Spectrum-based estimation of peak seismic responses}

For pounding caused by seismic loading, however, instead of eqn. (21), $\dot{u}_{\max }$ is estimated by using elastic design velocity spectrum $S_{p v}$ as follows 


$$
\dot{u}_{\max }=S_{p v}\left(T_{e q}, \xi_{e q}\right)=D_{\xi} \cdot S_{p v}\left(T_{e q}, \xi_{n p}\right), \quad D_{\xi}=\sqrt{\left(1+\alpha \xi_{n p}\right) /\left(1+\alpha \xi_{e}\right)}
$$

in above expressions, $D_{\xi}=$ scaling factor for taking into account the effect of damping, and $\alpha$ depends on each particular earthquake (table 2).

Peak collision force is approximated by using the SRSS rule for peak forces developed in contact element's spring and dashpot (fig. 1). Accordingly,

$$
\begin{aligned}
& F_{c}^{+}=\sqrt{\left[k_{s}^{+}\left(u^{+}-s^{+}\right)\right]^{2}+\left[c_{s}^{+}\left(\pi / \Delta t^{+}\right) u^{+}\right]^{2}\left[1-\left(s^{+} / u^{+}\right)^{2}\right]} \\
& F_{c}^{-}=\sqrt{\left[k_{s}^{-}\left(u^{-}-s^{-}\right)\right]^{2}+\left[c_{s}^{-}\left(\pi / \Delta t^{-}\right) u^{-}\right]^{2}\left[1-\left(s^{-} / u^{-}\right)^{2}\right]}
\end{aligned}
$$

\subsection{Validation of the method}

The validation study includes both one-side pounding and two-side pounding that considers same contact elements on both sides. For each pounding incident, it uses 8 systems with different $T_{n p}$ (table 1) but having same $\xi_{n p}=0.02$. For each system, separation distances are varied through 10 values of separation ratios $s / u_{n p}=0.1$ to 1.0 at an increment of 0.1 . Two different cases of contact element stiffness are considered: in stiff contact case the contact element is much stiffer than the system's stiffness; while in soft contact case the contact element is assumed quite flexible such that $\kappa=4$ for all systems (table 1). For each analysis case above, dashpot of contact element is chosen to simulate three values of coefficient of restitution, $e=0.9,0.6$ and 0.2 .

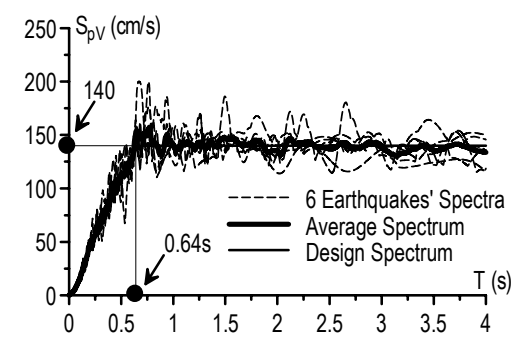

Figure 5: Spectra of artificial earthquakes and the design spectrum.

Table 1: Systems used in validation study.

\begin{tabular}{|c|c|c|c|c|c|c|c|c|c|}
\cline { 2 - 11 } \multicolumn{1}{c|}{} & System & $\mathbf{1}$ & $\mathbf{2}$ & $\mathbf{3}$ & $\mathbf{4}$ & $\mathbf{5}$ & $\mathbf{6}$ & $\mathbf{7}$ & $\mathbf{8}$ \\
\cline { 2 - 10 } \multicolumn{1}{c|}{} & $T_{n p}(\mathrm{~s})$ & 0.4 & 0.8 & 1.2 & 1.6 & 2.0 & 2.4 & 2.8 & 3.2 \\
\hline $\begin{array}{c}\text { Stiff } \\
\text { Contact }\end{array}$ & $T_{p d}(\mathrm{~s})$ & 0.1 & 0.16 & 0.2 & 0.2 & 0.2 & 0.24 & 0.28 & 0.32 \\
\cline { 2 - 10 } Soft & $\kappa$ & 16 & 25 & 36 & 64 & 100 & 100 & 100 & 100 \\
\hline $\begin{array}{c}\text { Contact } \\
\text { Cod }\end{array}$ & $\kappa$ & 0.2 & 0.4 & 0.6 & 0.8 & 1.0 & 1.2 & 1.4 & 1.6 \\
\hline
\end{tabular}

Table 2: Values of $\alpha$.

\begin{tabular}{|c|c|}
\hline Earthquake & $\boldsymbol{\alpha}$ \\
\hline Hachinohe EW & 75 \\
\hline JMA Kobe NS & 25 \\
\hline Tohoku NS & 75 \\
\hline El Centro NS & 55 \\
\hline Taft N111E & 75 \\
\hline BCJ-L2 & 75 \\
\hline
\end{tabular}


For time-history analysis, 6 artificial earthquakes (table 2) are considered in both positive and negative directions. These level 2 earthquakes have similar spectrum characteristics and similar to the elastic design spectrum. Thus, results obtained from numerical analysis can be used for investigating the accuracy of the proposed method. Fig. 5 shows the pseudo velocity spectra of these earthquakes ( $2 \%$ damping ratio), and the design spectrum having $S_{p v}=140 \mathrm{~cm} / \mathrm{s}$ in the constant velocity domain.

The accuracy of the proposed method in estimating peak displacement is demonstrated through ratios between estimated and respective time-history analysis results. The graphs in fig. 6 show the mean and standard deviation of these ratios vs. separation ratios, for each value of coefficient of restitution $e$. For one-side pounding, fig. 6a shows very close results between time-history analysis and estimation for $u^{+}$, ratios for $u^{-}$are slightly scattered at $s / u_{n p}$ small but most of them still distributes near 1.0. Accuracy of the method does not significantly change between soft and stiff contact case. Similarly to results of one-side pounding, the proposed method predicts very well peak displacement of symmetric two-side pounding (fig. 6b).

(a) One-Side Pounding

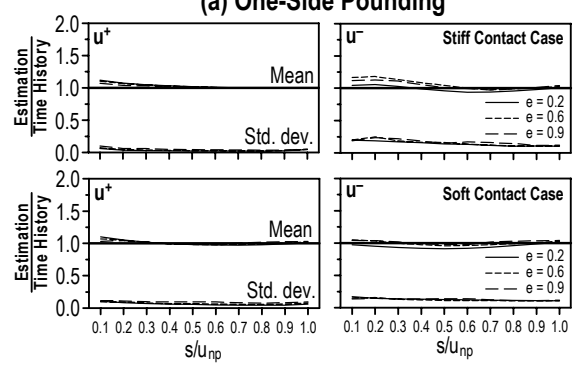

(b) Two-Side Pounding

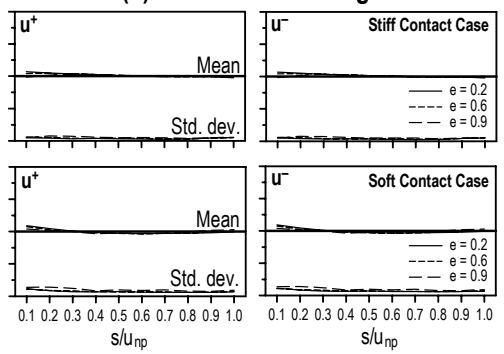

Figure 6: Accuracy of estimated peak displacement.

Peak collision force is normalized with the respective peak shear force that would develop in the system if pounding did not occur (i.e. $k \cdot u_{n p}$ ). Such normalized forces estimated by the proposed method are plotted against the accurate time-history analysis result in fig. 7, where each data point corresponds to one analysis case. These graphs show good estimation, especially for pounding with small coefficient of restitution and soft contact element. This can be understood because contact element with large damping can effectively dissipate energy, while soft contact element reduces collision force.

\section{Conclusions}

This paper proposed the new spectrum-based estimation method for peak structural responses of a SDOF system pounding against rigid structures. The pounding phenomenon is modeled using linear viscoelastic elements. This idealization is consistent with SDOF system used and appears adequate for studying the effects of pounding on the overall building responses. The 
spectrum-based estimation method is based on analytical solution of free vibration and harmonic vibration of the system both involving pounding to obtain equivalent period and damping, and uses them together with elastic design spectrum for estimation of peak responses. Using 6 artificial earthquakes that resemble the elastic design spectrum, the method is validated through extensive numerical experiments by varying system vibration period, separation distance, and contact element properties. The applicability of the method is shown and the correlation of estimated peak responses to time-history analysis results is very satisfactory.

The proposed method will serve as a basis that will be extend for structural pounding between two SDOF systems, as well as pounding of MDOF systems.

(a) One-Side Pounding

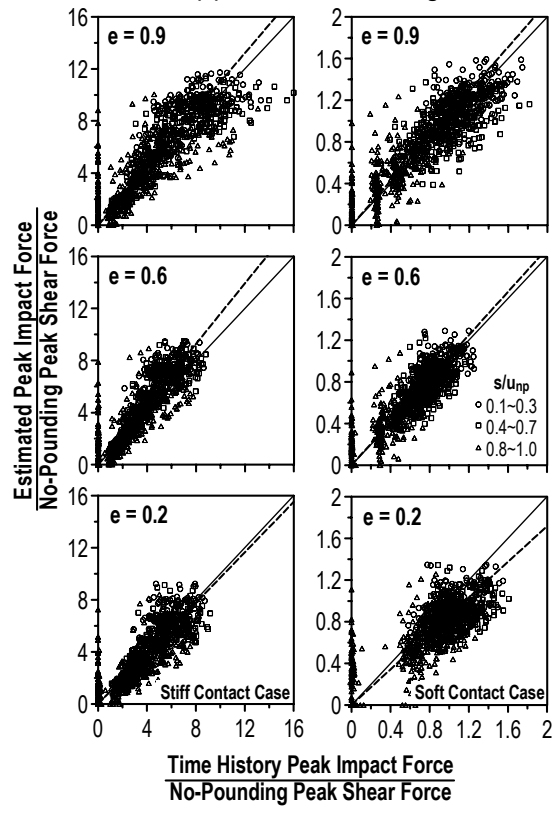

(b) Two-Side Pounding

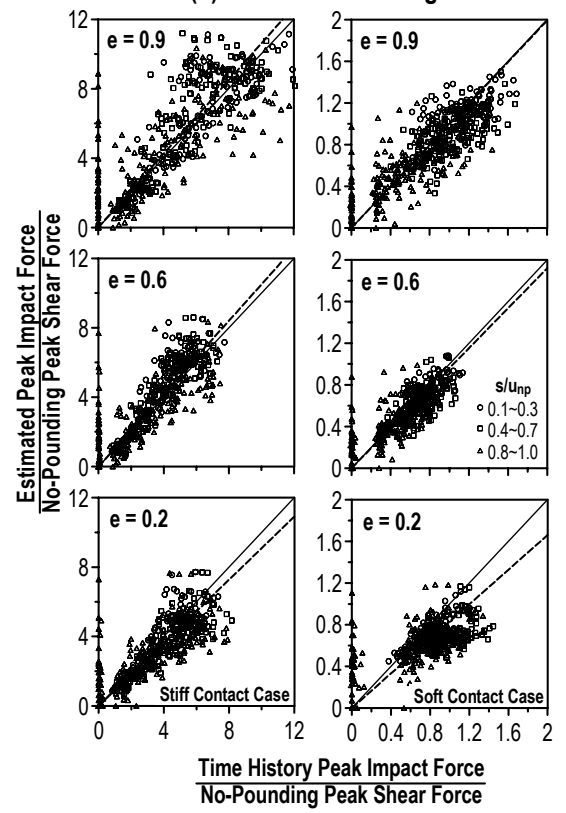

Figure 7: Accuracy of estimated peak collision force.

\section{Acknowledgement}

The first writer gratefully acknowledges the Japan Society for the Promotion of Science (JSPS) for giving him generous financial support to conduct researches in Japan as a JSPS post-doctoral fellow.

\section{References}

[1] Kasai, K. \& Tran, T.B., A simplified method to predict peak value and trend of seismic relative motion between adjacent buildings. J. Struct. Constr. Eng., AIJ, (582), pp. 47-55, 2004. 
[2] Kasai, K. \& Tran, T.B., Simplified method to predict relative motion between two inelastic structures subjected to time-lagged base motions. $J$. Struct. Constr. Eng., AIJ, (594), pp. 75-83, 2005.

[3] Wolf, J.P. \& Skrikerud, P.E., Mutual pounding of adjacent structures during earthquakes. Nuclear Eng. Design, (57), pp. 253-275, 1980.

[4] Anagnostopoulos, S.A., Pounding of buildings in series during earthquakes. Eq. Eng. Struct. Dyn., (16), pp. 443-456, 1988.

[5] Chau, K.T. \& Wei, X.X., Pounding of structures modeled as non-linear impacts of two oscillators. Eq. Eng. Struct. Dyn., (30), pp. 633-651, 2001.

[6] Weaver, W. JR., Timoshenko, S.P. \& Young, D.H., Vibration Problems in Engineering, $5^{\text {th }}$ Ed., John Wiley \& Sons: US, pp. 175-182, 1990.

[7] Anagnostopoulos, S.A., Equivalent viscous damping for modelling inelastic impacts in earthquake pounding problems. Eq. Eng. Struct. Dyn., (33), pp. 897-902, 2004. 